\title{
El suministro de energía eléctrica. Participación de las cooperativas en la gestión de este servicio de interés general
}

\author{
Vega María Arnáez Arce*
}

Sumario: I. Introducción. II. La externalización de la actuación de las administraciones públicas. II.1. Significado y alcance. II. 2. La prestación de servicios de interés general mediante personas jurídicas de base privada. III. Régimen jurídico del suministro de electricidad. III.1. Evolución de la regulación del sector eléctrico: del servicio público al servicio de interés general. III. 2. La separación de actividades en la nueva LSE: el suministro de energía eléctrica. a. Definición de la actividad de suministro b. Requisitos de los agentes comercializadores. IV. Las cooperativas eléctricas. IV.1. Breve aproximación al origen y evolución de las cooperativas eléctricas. IV.2. Aproximación al marco jurídico básico de las Cooperativas eléctricas. IV.3. Reconocimiento normativo y jurisprudencial de las cooperativas eléctricas como sociedades comercializadoras de electricidad. V. A modo de conclusión. Vı. Bibliografía.

Resumen: En este trabajo se analiza el régimen jurídico básico de las cooperativas eléctricas en su naturaleza jurídica de cooperativas de consumo que tienen por objeto social la distribución de la energía eléctrica y la comercialización de todo tipo de productos energéticos relacionados con la electricidad. Dicho análisis parte de la importancia cuantitativa y cualitativa de las cooperativas eléctricas, que representan en la actualidad un modelo de gestión eficiente que les permite garantizar el abastecimiento de electricidad tanto a sus socios como a cada vez un mayor número de clientes a precios muy económicos y competitivos, mediante el desarrollo de acuerdo con los principios y procedimientos de actuación propios de lo que se puede denominar un modelo energético sostenible.

Palabras clave: Desregulación, simplificación y externalización; servicios de interés general; suministro, distribución y comercialización de electricidad;

* Doctora en Derecho. Profesora de Derecho Administrativo de la Facultad de Derecho. Universidad de Deusto. Email: vegamaria.arnaez@deusto.es 
concurrencia competitiva; cooperativas de consumo eléctricas; ahorro y eficiencia energética.

Abstract: In this paper we analyze the basic legal regime of electric cooperatives in the legal nature of consumer cooperatives whose purpose is the distribution of electric energy and the commercialization of all types of energy products related to electricity. This analysis is based on the quantitative and qualitative importance of electric cooperatives, which nowadays represent an efficient management model that allows them to guarantee electricity supply to both their partners and to more and more customers at very low prices and Competitive, through the development in accordance with the principles and procedures of action of what can be called a sustainable energy model.

Keywords: Deregulation, simplification and outsourcing; Services of general interest; Supply, distribution and commercialization of electricity; Competitive concurrence; Electric consumer cooperatives; Saving and energy efficiency. 


\section{Introducción}

A lo largo de su evolución, el Derecho administrativo se ha configurado como el sector del ordenamiento jurídico que tiene por objeto la atribución de diversas potestades y prerrogativas de las Administraciones Públicas, al amparo de su función o misión de servicio a la garantía y realización de los intereses generales.

De igual modo, y como contrapeso del haz de las Potestades atribuidas a las Administraciones Públicas, el ordenamiento administrativo ha ido reconociendo y atribuyendo una sucesión de garantías de los administrados que, con el paso de tiempo, han ido creciendo tanto cuantitativa como cualitativamente como resorte para hacer frente a los poderes exorbitantes que implica el ejercicio de las funciones públicas.

En definitiva, el Derecho administrativo se puede definir como un derecho de prerrogativas y garantías en el que el ejercicio de las Potestades administrativas está sometido a una sucesión de principios y exigencias con la finalidad de asegurar la actuación objetiva de los poderes públicos en el ejercicio de sus funciones y competencias siempre al servicio de la realización del interés general.

Sin embargo, y aún cuando cualquier manifestación de las actuaciones de los Poderes Públicos es el resultado de un previo procedimiento administrativo concebido como una garantía para los administrados, lo cierto es que su tramitación evidencia una ralentización en la gestión de los intereses generales y en la prestación de los servicios públicos que lleva a cuestionar su virtualidad como garantía de los intereses de los ciudadanos.

De este modo, lo que está previsto y concebido en nuestro ordenamiento jurídico como una garantía de los administrados se llega a convertir en una auténtica traba que dificulta la gestión de los servicios públicos en los términos y con la diligencia y eficacia deseable para dar respuesta a las demandas y necesidades de los mismos.

Como reacción a esta situación, se plantea el fenómeno de la huida del derecho administrativo, o lo que es lo mismo, de la creación de una ficción jurídica consistente en la externalización de la gestión de los intereses y los servicios públicos a través de personas jurídicas o de organizaciones de base privada, bajo la forma jurídica de sociedades mercantiles (Gamero, 2013), en unos casos, o como instituciones sujetas a un régimen jurídico, organizativo y de funcionamiento propio y particular como el de las Entidades colaboradoras, en otras. 


\section{La externalización de la actuación de las administraciones públicas}

\section{II.1. Significado y alcance}

En la actualidad, la simplificación o desburocratización de la organización y del funcionamiento de las Administraciones Públicas se reconoce como uno de los retos más importantes y el objetivo más inmediato del Derecho administrativo, esencial para la construcción de un nuevo modelo de Administración Pública que garantice el servicio objetivo a los intereses generales y la realización efectiva -en cuanto eficaz y eficiente- de los servicios públicos, acorde con los principios constitucionales de la organización y la actuación administrativas proclamados en el Artículo 103 de nuestra Norma Fundamental (Palomar, 2010).

En este sentido, merecen significarse las reflexiones del profesor SEBASTIÁN MARTÍN-RETORTILLO calificando la simplificación administrativa como una necesidad inmediata de nuestras Administraciones Públicas que "[...] se enuncia siempre con un significado dinámico y operativo; en definitiva, como tarea a realizar que, según se verá, se proyecta en ámbitos muy distintos. En todo caso, y como punto de partida, parece obligado reconocer la necesidad de llevarla a cabo. Es algo que no cabe cuestionar; tampoco que haya que hacerlo en términos rigurosos y que no admiten demora [...]»(MartínRetortillo, 1998:7).

Así pues, el fenómeno de la externalización de la actuación administrativa se configura en el Derecho administrativo actual como una tendencia imparable de transformación de las Administraciones Públicas que, impulsada por las corrientes doctrinales de la denominada nueva gestión pública, facilita y favorece la incorporación de los conocimientos técnicos, así como de las destrezas y habilidades materiales, imprescindibles para garantizar una gestión y una prestación eficaz y eficiente de los servicios públicos.

En definitiva, la gestión de los servicios públicos mediante personas jurídicas privadas se ha convertido en un elemento clave de la transformación de la organización y el funcionamiento de las Administraciones Públicas de nuestros días, dirigido a materializar y consagrar la realización del Principio constitucional de eficacia de la actuación administrativa (Sosa, 2002). 


\section{2. La prestación de servicios de interés general mediante personas} jurídicas de base privada

A lo largo de la historia y en el camino de su evolución hacia la desregulación o simplificación, se ha generalizado el fenómeno de la externalización de la gestión y prestación de los servicios de interés general mediante el recurso a fórmulas societarias o personas jurídicas de base privada.

La externalización de la actuación de las Administraciones Públicas ha provocado la pérdida del carácter orgánico del concepto de servicio público que pasa a configurarse y definirse a partir su elemento sustantivo o material, relativo a los aspectos o a las técnicas jurídicas propias del Derecho Privado y que son gestionadas tanto por los Poderes Públicos como por los particulares (Sosa, 2002).

Resulta cada vez más frecuente y habitual que los sujetos privados, cuya única relación con las Administraciones Públicas hasta ahora consistía en ser receptores de su actuación en cuanto destinatarios de los servicios públicos, empiecen a desarrollar funciones esencialmente administrativas, ejerciendo funciones y Potestades públicas similares a las que ostentan las Administraciones Públicas.

En este sentido, señala SANTAMARÍA PASTOR que el «el hecho de que personas y organizaciones privadas realicen funciones públicas y ostenten, a tal fin, poderes también inequívocamente públicos, no es una anomalía. En todos los tiempos y países, los Estados han asumido un conjunto de responsabilidades muy superior a las que podían desempeñar con su propio personal y han tenido que confiar parte de ellas a personas y organizaciones privadas, bien que sometidas en este aspecto a un régimen público y al control, más o menos intenso, de las Administraciones titulares de dichas funciones y poderes» (Santamaría, 2009:37).

Sin embargo, el problema se ha planteado, al igual que ha sucedido en el ámbito de la Administración Instrumental, cuando la encomienda del ejercicio de funciones públicas o de prestación de servicios públicos a través de personas jurídicas de base privada se ha ido improvisando y construyendo de forma empírica, sin la previa existencia de una tipificación legal común de todos los supuestos en que tiene lugar, de tal forma y manera que sus concretas características y régimen jurídico es en cada caso diferente (Santamaría, 2009).

Merece significarse a este respecto la sistematización, elaborada por el profesor SANTAMARÍA PASTOR, de los tres modelos básicos en virtud de los cuales se ha venido concretado la encomienda de gestión de los intereses y servicios públicos a los particulares. 
Concretamente en los siguientes términos:

«a) En primer lugar, un conjunto de entidades asociativas que agrupan a sujetos privados pertenecientes a un colectivo profesional o empresarial. Estas entidades no se constituyen en virtud de pactos privados (como una asociación civil), sino por un acto del poder público, generalmente amparado en una ley previa, de manera que no poseen una forma de personificación asimilable a las existentes en el derecho privado, sino pública y singular: nos referimos a las que genéricamente son conocidas como Corporaciones de Derecho Público (categoría que engloba un conjunto muy variado de entidades: Colegios Profesionales, Cámaras de Comercio, Industria y Navegación, Cámaras Agrarias; Cofradías de Pescadores, Comunidades de Usuarios de recursos hidráulicos y Federaciones Deportivas, entre otras).

b) En segundo lugar, las empresas privadas (normalmente Sociedades Mercantiles) que prestan un servicio público administrativo en virtud de un contrato con la Administración titular del mismo; contrato cuyas cláusulas definen sus respectivos derechos y obligaciones. Por lo general, estas entidades reciben el nombre de concesionarios.

c) $Y$, por fin, un amplio y heteróclito conjunto de empresas privadas que, por encomienda expresa de la Ley o en virtud de otros títulos habilitantes (autorizaciones), realizan funciones administrativas no calificables como servicios públicos (p.ej. actividades de inspección como sucede en el ámbito de la seguridad industrial) o prestan servicios de interés general y de carácter fundamental para el correcto funcionamiento de un sector [...]» (Santamaría, 2009:37-39).

El fundamento del recurso a estas formas indirectas de prestación de servicios públicos no es otro sino la remisión genérica a las normas de derecho privado, civil o mercantil, para la determinación del régimen jurídico de actuación y de funcionamiento de estas sociedades en cuanto se refiere a su constitución, organización y funcionamiento, de tal forma y manera que la aplicación de normas propias y específicas de derecho administrativo se reduce o limita exclusivamente a los supuestos en que éstas sociedades actúan en ejercicio de potestades o prerrogativas públicas, ejerciendo a tal efecto funciones públicas (García, 2013; Aguilar Rubio-Vargas Vasserot, 2012; Vargas Vasserot, 2012; Santamaría, 2009; Cosculluela, 2009; Parada, 2010; García de Enterría, 2002).

Las cooperativas de consumo eléctricas forman parte de esta categoría de personas jurídicas de base privada, en cuanto tienen por objeto social la prestación de servicios de interés general esenciales y determinantes del correcto funcionamiento del sector eléctrico (Sosa, 2008). 
En este sentido, se configuran como auténticos operadores del sistema a los que les corresponde la realización de cuantas actividades sean necesarias para garantizar tanto la producción como la continuidad en el suministro eléctrico, así como la coordinación de los sistemas de transporte, distribución y comercialización de la electricidad.

\section{Régimen jurídico del suministro de electricidad}

\section{1. Evolución de la regulación del sector eléctrico: del servicio público al servicio de interés general}

La regulación del sector eléctrico, como de tantos otros sectores de actuación de las administraciones públicas ha sido el resultado de la experiencia, de tal forma y manera que se puede afirmar que en el origen y la evolución de la normativa vigente concurren un conjunto de factores sociales, tecnológicos, económicos, empresariales y ambientales específicos.

El sector eléctrico se caracteriza por ser un sector estratégico y de interés general por su objeto, la energía eléctrica, cuya distribución y suministro se debe garantizar a todos los ciudadanos, intensificando para ello su regulación hasta modelos monopolísticos centralizados, en tanto que se trata de un bien jurídico indispensable para el desarrollo de la vida cotidiana y determinante del crecimiento económico (González-Varas, 2001).

La evolución de la normativa del sector eléctrico forma parte de un proceso de transformación de la regulación de los grandes servicios colectivos, como las telecomunicaciones, el gas y los transportes, entre otros, caracterizado por la sustitución del tradicional concepto de servicio público, concebido como servicio de titularidad y gestión públicas y sujeto a normas de derecho administrativo, por un modelo liberalizado en el que se introducen el mercado y la libre competencia en la prestación de los referidos servicios colectivos (Hernández, 2005).

La introducción de este nuevo modelo participativo y de concurrencia competitiva en el sector eléctrico ha sido especialmente compleja por la concurrencia de condicionantes de técnicos y económicos que determinantes de la tendencia al mantenimiento del monopolio del sector en todas sus fases desde la generación o producción de electricidad hasta su distribución y comercialización.

La regulación competitiva del sector eléctrico en nuestro país se produjo a partir de la aprobación y entrada en vigor de la Ley 54/1997, de 27 de noviembre, del Sector Eléctrico, en el marco de la desregula- 
ción como reto del Derecho administrativo en su proceso de transformación y adaptación a las nuevas realidades tecnológicas, ambientales, económicas y sociales.

Este cambio en la regulación del sector eléctrico coincidió con la redefinición del concepto de servicio público para abrirse progresivamente a la competencia y al mercado.

Se pasó, de este modo, desde la reserva a favor del Estado de la titularidad, gestión y regulación de los servicios públicos a la definición y el establecimiento de un conjunto de actividades y obligaciones que se imponen a los particulares que ostenten la condición de prestadores de un servicio de interés general, que tienen por objeto garantizar la universalidad y la efectividad de su prestación (Bacigalupo, 2009).

La liberalización del sector eléctrico se presentó, por tanto, como un proceso decisivo que tuvo su origen en la incorporación de España a la Comunidad Económica Europea y que se materializó mediante su definición y caracterización como servicio de interés general prestado en régimen de libre competencia.

De este modo, se produjo la desintegración vertical de las distintas actividades, segregando las actividades desarrolladas en régimen de monopolio natural, cuales son el transporte y la distribución de electricidad, de aquéllas que se desarrollan en régimen de libre concurrencia, esto es, las de su generación, comercialización o suministro.

Transcurridos dieciséis años desde entrada la entrada en vigor de la precitada Ley 54/1997, el pasado 27 de diciembre de 2013 se publicó en el Boletín Oficial del Estado la nueva Ley 24/2013, de 26 de diciembre, del Sector Eléctrico (en adelante LSE), en cuya Exposición de Motivos se proclama como su finalidad básica la de «[...] establecer la regulación del sector eléctrico garantizando el suministro eléctrico con los niveles necesarios de calidad y al mínimo coste posible, asegurar la sostenibilidad económica y financiera del sistema y permitir un nivel de competencia efectiva en el sector eléctrico, todo ello dentro de los principios de protección medioambiental de una sociedad moderna $[\ldots]$ ».

Se debe apuntar a este respecto que esta nueva LSE se enmarca en un contexto de integración de los mercados eléctricos europeos, teniendo en cuenta la normativa europea de aplicación en el sector eléctrico, en particular, la Directiva 2009/72/CE del Parlamento Europeo y del Consejo de 13 de julio de 2009 sobre normas comunes para el mercado interior de la electricidad, que fue traspuesta por el Real Decreto-Ley 13/2013, de 30 de marzo. También tiene en cuenta el marco europeo de intercambios transfronterizos de electricidad establecido 
por el Reglamento (CE) número 714/2009, del Parlamento Europeo y del Consejo, de 13 de julio de 2009, regulador de las condiciones de acceso a la red para el comercio transfronterizo de electricidad.

\section{2. La separación de actividades en la nueva LSE: el suministro de energía eléctrica}

La nuevo LSE, en su artículo 1 define su objeto como el del establecimiento de la regulación del sector eléctrico con la finalidad de garantizar el suministro de energía eléctrica, y de adecuarlo a las necesidades de los consumidores en términos de seguridad, calidad, eficiencia, objetividad, transparencia y, todo ello, al mínimo coste posible.

A tal efecto, califica como actividades destinadas al suministro de energía eléctricas las de generación, transporte, distribución, servicios de recarga energética, comercialización e intercambios intracomunitarios e internacionales, así como la gestión económica y técnica del sistema eléctrico.

Y así, mediante la regulación de dichas actividades el legislador pretende adecuar el suministro eléctrico a las necesidades de los consumidores, en base a criterios de eficiencia, racionalización y optimización de los recursos disponibles.

Para ello, la LSE establece dos ámbitos objetivos y diferenciados en su regulación distinguiendo las actividades del sector eléctrico que son propiamente competitivas como la producción, la generación y el suministro o comercialización de la electricidad, de las actividades reguladas como el transporte y la distribución.

Nos centraremos en el análisis del régimen jurídico de una de las actividades competitivas, el suministro de energía eléctrica, regulado en el Título VIII, artículos 43 a 60 de la vigente LSE.

\section{a. DefINICIÓN DE LA ACTIVIDAD DE SUMINISTRO}

La actividad de suministro de la energía eléctrica, al igual que la producción, es una actividad expresamente calificada por la LSE como actividad competitiva o liberalizada que se encuentra sometida, no obstante, a determinadas medidas reguladoras que tienen por objeto garantizar el suministro efectivo de electricidad y organizar el funcionamiento efectivo del mercado eléctrico.

De acuerdo con lo dispuesto en el artículo 43 de la LSE, el suministro de energía eléctrica se puede definir como la entrega de energía a través de las redes de transporte y distribución mediante contrapresta- 
ción económica en las condiciones de regularidad y calidad que resulten exigibles.

La comercialización de electricidad se configura en la LSE como una actividad que se presta o realiza en régimen de libre competencia basada en los principios de libertad de entrada y acceso a las redes y libertad de contratación y formación competitiva de los precios (Hernández, 2005).

De este modo, la LSE impulsa el principio de competencia efectiva en el sector eléctrico, introduciendo un mecanismo competitivo para la reducción del poder de monopolio en los mercados de restricciones, un nuevo régimen de regulación de las energías renovables, de cogeneración y residuos que reduce las distorsiones que estas creaban en el mercado y las hace partícipes del mismo en cuanto a que su retribución no será ajena al mercado, un aumento de las competencia en las comercializadoras de referencia, la mejora de la posición del consumidos en cuanto a información disponible y facilitación de procesos de cambio de suministrador, entre otros.

No obstante, la libre entrada o el libre acceso al mercado de la comercialización de la electricidad sigue supeditada al cumplimiento por el sujeto comercializador de los tres requisitos siguientes: autorización administrativa o comunicación previa de inicio de actividad y declaración responsable, inscripción en el registro administrativo de distribuidores, comercializadores y consumidores y prestar caución o garantía para atender a las demandas de energía.

\section{b. REQUISITOS DE LOS AGENTES COMERCIALIZADORES}

De acuerdo con lo dispuesto en el artículo 6.1 f) de la LSE, tendrán naturaleza de las entidades comercializadoras de energía eléctrica aquellas sociedades mercantiles, o sociedades cooperativas de consumidores y usuarios, que, accediendo a las redes de transporte o distribución, adquieren energía para su venta a los consumidores, a otros sujetos del sistema o para realizar operaciones de intercambio internacional en los términos legalmente establecidos.

En este sentido, debe ponerse de manifiesto la vigencia y aplicación al supuesto que nos ocupa de la Ley 17/2009, de 23 de noviembre, sobre el libre acceso a las actividades de servicios y su ejercicio, por la que se incorpora al derecho español la Directiva 2006/123/CE del Parlamento Europeo y del Consejo, de 12 de diciembre de 2006, relativa a los servicios en el mercado interior, en adelante Directiva de servicios.

Esta Ley 17/2009 se incorporó a nuestro ordenamiento jurídico con el propósito de consolidar los principios reguladores compatibles con 
las libertades básicas de establecimiento y de libre prestación de servicios y suprimir las barreras o trabas que restringen el acceso a las actividades de servicios y su ejercicio.

En particular, para que los instrumentos de intervención de las Administraciones Públicas en este sector respetaran los principios de no discriminación, de justificación por razones imperiosas de interés general y de proporcionalidad para atender esas razones.

Aún más, dicha norma exige que se simplifiquen los procedimientos, evitando dilaciones innecesarias y reduciendo las cargas administrativas a los prestadores de servicios. Adicionalmente, se refuerzan las garantías de los consumidores y usuarios de los servicios, al obligar a los prestadores de servicios a actuar con transparencia tanto respecto a la información que deben proveer en materia de reclamaciones.

Pues bien, con el fin de alcanzar el objetivo de reformar el marco regulador del acceso a las actividades de servicios ha sido necesario un ejercicio de evaluación y adaptación de toda la normativa sectorial vigente para adecuarla a los fines y objetivos que establece la Ley $17 / 2009$.

En este contexto se ha aprobado la Ley 25/2009, de 22 de diciembre, conocida como Ley Ómnibus, de modificación de diversas leyes para su adaptación a la Ley sobre el libre acceso a las actividades de servicios y su ejercicio para adaptar la normativa estatal de rango legal a lo dispuesto en la precitada Ley 17/2009.

En cuanto se refiere al sector eléctrico, la adaptación de la normativa a los nuevos requerimientos se ha producido a partir de la aprobación del Real Decreto 198/2010, de 26 de febrero, que tiene por objeto el desarrollo de los preceptos de la vigente LSE modificados por la Ley 25/2009, de 22 de diciembre.

Respecto de la regulación de la actividad de comercialización de la electricidad, el artículo 3 del precitado Real Decreto 198/2010, introduce importantes modificaciones, entre las que destacamos especialmente las dos siguientes:

1. ${ }^{a}$ Respecto de las obligaciones de las empresas comercializadoras se sustituye la autorización administrativa previa por una comunicación de inicio de actividad que deberán presentar ante la Dirección General de Política Energética y Minas del Ministerio de Industria, de acuerdo con el modelo establecido en el anexo 6.1 del Real Decreto 198/2010 y en la que se debe especificar el ámbito territorial en el que se vaya a desarrollar la actividad. El interesado debe acompañar a la comunicación de inicio de actividad una declaración responsable sobre el cumplimiento de 
los requisitos que exige la legislación vigente para la comercialización de la electricidad.

Se impone asimismo al interesado la obligación de comunicar cualquier circunstancia modificativa de los datos incluidos tanto en la comunicación de inicio de actividad como en la declaración responsable originaria.

2. ${ }^{a}$ Por otra parte, el Real Decreto 198/2010 modifica los requisitos necesarios para realizar la actividad de comercialización, acreditativos de la capacidad legal, técnica y económica de las empresas comercializadoras.

La acreditación de la capacidad legal de las empresas que realizan la actividad de comercialización de electricidad se formaliza mediante su configuración como sociedades mercantiles debidamente inscritas en el registro correspondiente.

Respecto de su capacidad técnica, dichas sociedades deben acreditar el cumplimiento de los requisitos exigidos por la LSE a los sujetos compradores en el mercado de producción de energía eléctrica conforme a los procedimientos de operación técnica $y$, en su caso, las reglas de funcionamiento y liquidación del mercado de producción.

Finalmente, la capacidad económica de las empresas interesadas en la comercialización de la electricidad se debe acreditar mediante la prestación de las cauciones o avales exigibles para la adquisición de energía en el mercado de producción de electricidad ante los operadores del sistema y del mercado.

\section{Las cooperativas eléctricas}

\section{IV.1. Breve aproximación al origen y evolución de las cooperativas eléctricas}

Durante la primera mitad del siglo xx surgen las primeras cooperativas eléctricas en nuestro país, con el objetivo de resolver el problema de la falta de suministro eléctrico en pequeños núcleos de población y para poner en marcha e impulsar el desarrollo de iniciativas industriales en un momento caracterizado por la ausencia de infraestructuras a través de las que garantizar el servicio eléctrico para toda la población.

Las cooperativas eléctricas tienen, por lo tanto, su origen en lugares apartados y barrios periféricos de las ciudades en los que no les interesaba invertir a las grandes mercantiles por la difícil amortización o ren- 
tabilización de las nuevas redes de distribución necesarias para garantizar el suministro de energía eléctrica en estos lugares.

Tras la Guerra Civil se experimenta un proceso de paulatina desaparición de estas sociedades cooperativas como consecuencia de las fuertes restricciones económicas derivadas de la postguerra y de la creciente presión ejercida por las grandes mercantiles interesadas por mantener y reforzar su presencia en un sector cada vez más monopolizado.

Sin embargo, las cooperativas eléctricas subsisten a lo largo del tiempo, existiendo en la actualidad varias sociedades que actúan como distribuidoras y como comercializadoras de energía eléctrica, que adquieren al por mayor en el mercado, repercutiendo el ahorro generado a sus socios en el momento de la comercialización.

En la actualidad existen en nuestro país numerosas cooperativas eléctricas, distribuidoras y comercializadoras, la mayoría emplazadas en la Comunidad Valenciana. Entre otras, Cooperativa Eléctrica de Castelar, Cooperativa Eléctrica Albaterense, Cooperativa Eléctrica Benéfica Catralense, Cooperativa Popular de Fluido Eléctrico de Camprodon, Cooperativa Valenciana Electrodistribuidora de Fuerza y Alumbrado Serrallo, Electrica Algimia de Alfara, Sociedad Cooperativa Valenciana, Electrica Vinalesa, S.C.V., Eléctrica de Chera, S.C.V., Eléctrica de Guadassuar, S.C.V., Eléctrica de Meliana, S.C.V., Eléctrica de Sot de Chera, S.C.V., Eléctrica del Pozo, Sociedad Cooperativa Madrileña, Eléctrica Nuestra Señora de Gracia, S.C.V., Eléctrica Popular, S. Cooperativa Madrileña, Electrodistribuidora de Fuerza y Alumbrado, S.C.V., Electroharinera Belsetana, Sociedad Cooperativa, Fluido Eléctrico de Museros, S.C.V., Grupo de electrificación rural de Binefar y comarca, S. Coop. R.L., Benéfica de consumidores de electricidad «San Francisco de Asís» de Crevillent, Suministros especiales Alginetenses, S.C.V.

Las cooperativas de consumo eléctricas centran su actividad en el mantenimiento de las redes de distribución de energía con la finalidad de mejorar la calidad del suministro y evitar así las pérdidas en la actividad de transporte y comercialización.

De este modo, las cooperativas pueden repercutir el ahorro generado a sus socios, ofreciendo precios muy competitivos y baratos en el mercado, alcanzando incluso niveles tarifarios similares al costo del bono social.

Al disponer de escasos recursos económicos que les permitan invertir en nuevas redes, las cooperativas eléctricas centran su actividad en áreas geográficas reducidas como pueblos, núcleos rurales y pequeños barrios en los que les resulta más fácil su desarrollo por la concentración de socios en la misma zona o área geográfica. 
En general, se puede afirmar que, en la actualidad, las cooperativas eléctricas representan un modelo de buena gestión que les permite suministrar energía eléctrica a un precio entre un $5 \%$ y un $7 \%$ más barato a sus socios y a un número creciente de clientes no socios. En este sentido, la Alianza Cooperativa Internacional ha reconocido el importante papel que desempeñan las sociedades cooperativas en relación con el ahorro y la eficiencia energética.

Debe significarse a este respecto, la contribución de las sociedades cooperativas al modelo de consumo y generación de energía eléctrica propugnado en la vigente Ley $2 / 2011$, de 4 de marzo, de Economía Sostenible, en adelante LES, compatible con la normativa y los objetivos comunitarios y con los esfuerzos internacionales en la lucha contra el cambio climático, en cuyo Capítulo I del Título III define el régimen jurídico de lo que denomina un modelo energético sostenible enunciando como Principios de la política energética los de Garantía de la seguridad del suministro; Eficiencia económica y Sostenibilidad ambiental.

A tales efectos, la LES (artículo 77.3) impone al Gobierno la obligación de impulsar la diversificación de las fuentes de suministro energético, el desarrollo eficiente de las infraestructuras y redes de transmisión, la transparencia y la competitividad de los mercados, la suficiencia de las retribuciones y la creciente incorporación de las energías renovables y las políticas de ahorro y eficiencia.

En este nuevo contexto, destaca el papel de las cooperativas de consumo distribuidoras y comercializadoras de electricidad, en tanto que desarrollan un importante trabajo por su contribución a la lucha contra el cambio climático, siendo destacables sus esfuerzos en la utilización de energías renovables, reconocidos expresamente por la Alianza Cooperativa Internacional².

\section{IV.2. Aproximación al marco jurídico básico de las Cooperativas eléctricas}

De acuerdo con la declaración formulada en el año 1995 por la Alianza Cooperativa Internacional, el fenómeno cooperativista está basado en los valores de la autoayuda, autorresponsabilidad, democracia, igualdad, equidad y solidaridad.

2 http://www.empresaytrabajo.coop, última consulta a 30 de julio de 2014. 
En la actualidad, se acepta mayoritariamente la definición del artículo 1 LCPV según la cual la cooperativa es una empresa que tiene por objeto prioritario la promoción de actividades económicas y sociales de sus miembros y la satisfacción de sus necesidades con la participación activa de los mismos, observando los principios cooperativos y atendiendo a la comunidad de su entorno (Gadea, 2009).

En definitiva, lo que distingue o singulariza a la entidad cooperativa de otras personas jurídicas con carácter empresarial no es el tipo o clase de actividad a desarrollar, sino los principios inspiradores y rectores de su estructura y funcionamiento: los principios cooperativos revisados periódicamente por la $\mathrm{ACl}$ que diferencian a las cooperativas de cualquier otro tipo de empresas y que se concretan en los siguientes: la adhesión voluntaria y abierta de sus miembros, la gestión democrática por los socios, participación económica de los socios, autonomía e independencia, educación, formación e información, cooperación entre cooperativas e interés por la comunidad (Gadea, 2009).

Tanto la Ley 27/1999, de 16 de julio de Cooperativas como la Ley 4/1993, de 24 de julio, de Cooperativas de Euskadi, definen las Sociedades Cooperativas como sociedades integradas por personas que se asocian de forma voluntaria con el fin de desarrollar en común una o varias actividades de carácter empresarial, acogiéndose a un modelo organizativo al servicio de sus miembros, cuyo principal objetivo es la promoción de las actividades económicas y sociales de aquellos, para el desarrollo de una empresa de propiedad conjunta y gestión democrática, con plena observancia y respeto de los principios cooperativos en su estructuración y funcionamiento.

Sea cual fuere la actividad cooperativizada debe servir a una función social, entendida como satisfacción de alguna de las necesidades de promoción de un interés de carácter socioeconómico de sus miembros que se anteponga al interés de un concreto y determinado beneficio económico.

La Ley Vasca regula las diferentes clases de cooperativas en el capítulo I de su Título II, especificando que éstas además de someterse a los principios configuradores establecidos en la ley, están sujetas a la legislación específica que resulte en cada caso aplicable, en función de cuál sea la concreta actividad que a la que se dediquen.

En el supuesto que nos ocupa, de la comercialización de la electricidad a través de sociedades cooperativas, la forma jurídica adecuada sería la de las cooperativas de consumo, en tanto que, de acuerdo con lo dispuesto en el artículo 105 de la Ley Vasca de Cooperativas tienen por objeto «procurar bienes o prestar servicios para el uso o 
consumo de sus socios y de quienes con ello convivan, así como la defensa y promoción de los derechos e intereses legítimos de los consumidores y usuarios. Pueden ser socios de estas cooperativas las personas físicas y las entidades $u$ organizaciones que tengan el carácter de destinatarios finales».

De este modo, las cooperativas eléctricas se constituyen como cooperativas de consumo que tienen por objeto la distribución de la energía eléctrica y la comercialización de todo tipo de productos energéticos relacionados con ella, en los términos previstos en la legislación vigente, así como la prestación de todos los servicios y actividades relacionados directa o indirectamente con dichas operaciones.

\section{IV.3. Reconocimiento normativo y jurisprudencial de las cooperativas eléctricas como sociedades comercializadoras de electricidad}

Tanto la legislación estatal como autonómica vigente en materia de cooperativas reconocen y afirman la personalidad jurídica de estas sociedades a partir de su inscripción en el Registro de Cooperativas, que tiene por objeto su calificación y clasificación conforme a los principios de publicidad, legitimación, privacidad y tracto sucesivo, gozando las inscripciones practicadas en sus libros de sendas presunciones de veracidad y exactitud.

Aún más, ambas leyes, tanto la Ley 27/1999, de 16 de julio de Cooperativas como la Ley 4/1993, de 24 de julio, de Cooperativas de Euskadi, en sus respectivos artículos primeros, afirman que cualquier actividad económica lícita podrá ser organizada y desarrollada mediante una sociedad constituida al amparo de estas normas.

Declaración que, para el supuesto específico de las cooperativas de consumidores y usuarios se reconoce expresamente en el Texto Refundido de la Ley General para la Defensa de los Consumidores y Usuarios aprobada por Real Decreto Legislativo núm. 1/2007, de 16 de noviembre.

Del mismo modo, el Tribunal Supremo de Justicia ha reconocido expresamente a las sociedades cooperativas como personas jurídicas legalmente habilitadas para el desarrollo de la actividad de comercialización de energía eléctrica, mediante Sentencia dictada con fecha 16 de octubre de 2003 (RJ [2003/9558], con ocasión de la impugnación de diversos preceptos del Real Decreto 1955/2000, en la que el Alto Tribunal declara ilegal la exigencia de que únicamente puedan ejercer la actividad de comercialización de la electricidad las sociedades inscritas en el Registro Mercantil. 
Por su parte, el reconocimiento normativo, en normas de derecho administrativo sectorial, de las sociedades cooperativas como comercializadoras de electricidad se produjo a través del Real Decreto 198/2010, de 26 de febrero, por el que se adaptan determinadas disposiciones relativas al sector eléctrico a lo dispuesto en la Ley 25/2009, de modificación de diversas leyes para su adaptación a la ley sobre el libre acceso a las actividades de servicios y su ejercicio.

En concreto, mediante la modificación del contenido del artículo 73 del Real Decreto 1955/2000, que en su nueva redacción, al enunciar los requisitos necesarios para el ejercicio de la actividad de comercialización de la electricidad, únicamente exigen que las empresas acrediten su capacidad mediante su inscripción en el correspondiente Registro Público, sin que existan más limitaciones o reservas.

Finalmente, y a diferencia de lo que ocurría en la legislación anterior, la nueva LSE ha reconocido expresamente a las sociedades cooperativas de consumidores y usuarios como comercializadores de energía eléctrica. Concretamente en el apartado f) del artículo 6.1.

\section{A modo de conclusión}

El camino hacia la simplificación y la progresiva desburocratización de la organización y de la actuación de las Administraciones Públicas se configura en la actualidad como uno de los retos más importantes y significativos del derecho administrativo de nuestro tiempo. En este nuevo contexto, cada vez más participativo, los sujetos privados, investidos de diversas personificaciones jurídicas asumen la gestión y prestación de diferentes servicios de interés general.

En el supuesto analizado del sector eléctrico, las cooperativas de consumo destacan por el ejercicio de las actividades de distribución y comercialización de energía eléctrica y otros productos energéticos relacionados a bajo coste, tanto a sus socios como, cada vez en más ocasiones, a terceros usuarios.

Las primeras cooperativas eléctricas surgieron en nuestro país como cooperativas de consumo que, a comienzos del siglo xx, se constituyeron en el contexto de pequeños núcleos de población. Tras la Guerra Civil sufrieron un proceso de reducción o limitación como consecuencia de las restricciones derivadas de la postguerra y de la presión creciente de las grandes mercantiles que consolidaban sus posiciones en un sector que caminaba hacía su configuración como uno de los grandes monopolios del Estado. 
Desde un punto de vista sustantivo o material, las cooperativas eléctricas se erigen como modelos a seguir por su trabajo relacionado con la búsqueda y experimentación de nuevos modelos de consumo y generación de energía eléctrica y por su compromiso en la lucha contra el cambio climático y con el ahorro energético, así como con la incorporación de energías renovables al proceso de producción de electricidad.

Por otra parte, y desde un punto de vista estrictamente formal, el reconocimiento de las cooperativas eléctricas como agentes comercializadores de energía se ha incorporado a nuestro ordenamiento jurídico a través del Real Decreto 198/2010, de 26 de febrero, por el que se modifica el artículo 73 del Real Decreto 1955/2000, de 1 de diciembre, por el que se regulan las actividades de transporte, distribución, comercialización, suministro de electricidad, junto con los procedimientos de autorización de instalaciones de energía eléctrica.

En definitiva, se puede concluir que las cooperativas de consumo distribuidoras y comercializadoras de electricidad representan, en la actualidad, un modelo de eficiencia y buena gestión en la prestación del servicio de interés general consistente en el suministro de electricidad, que les permite garantizar el abastecimiento de energía tanto a sus socios como a cada vez un mayor número de clientes, a precios más económicos y competitivos y en mérito y base a principios y procedimientos de actuación propios de un modelo energético sostenible, destacando la obra social desarrolla a este respecto por estas sociedades.

\section{Bibliografía}

Aguilar Rubio, M y Vargas Vasserot, C. (2012) Las cooperativas sin ánimo de lucro en la prestación de servicios públicos. Análisis de fiscalidad, Sociedad y Utopía, núm. 40, 2012, p. 404-435.

Arenilla Sáez, M. (2011) Crisis y reforma de la Administración Pública, La Coruña, Netbiblo.

Argudo Périz, J.L. (2007) Las cooperativas sin ánimo de lucro: ¿vuelta a los orígenes o respuesta a las nuevas necesidades sociales, Revista Vasca de Economía Social, N. ${ }^{\circ}$ 3, p. 179-201.

Arnázz ArCe, V.M. (2011) La comercialización de energía eléctrica a través de cooperativas de consumo, Boletín de la Asociación Internacional de Derecho Cooperativo, N. ${ }^{\circ} 45$, p. 197-216.

Arnáez ArCe, V.M. (2012) La participación del cooperativismo en las políticas públicas, Boletín de la Asociación Internacional de Derecho Cooperativo, N. ${ }^{\circ} 46$ p. $185-200$. 
Arnáez Arce, V.M. (2013) La potestad planificadora de las Administraciones Públicas, Bilbao, Gomylex.

Arnáez Arce, V.M. y Atxabal Rada, A. (2014) Las cooperativas de utilidad pública e iniciativa social. Aspectos administrativos y fiscales, Boletín de la Asociación Internacional de Derecho Cooperativo, N. ${ }^{\circ} 47$, p. 199-228.

BACIgALUPO SAGGESE, M. (2009) electricidad, servicio público y mercado en Tratado de regulación del sector eléctrico, TOmo / BECKOR, F; CAZORLA, L.M.; MARTínez-SimAnCAS, J. y SALA, J.M. (Dirs), Pamplona, Thomson-Aranzadi, 2009, p. 76-93.

Bermejo Vera, J. (1995). Constitución y planificación hidrológica, Madrid, Civitas.

Bermejo Vera, J. y Fernández Farreres, G. (1982) Actuaciones Administrativas aisladas versus planificación: modelo urbanístico y protección del patrimonio histórico-artístico, Cuadernos Aragoneses de Economía, N. ․ 1981/1982.

Coque Martínez, J. (2005) Compartir soluciones: las cooperativas como factor de desarrollo en zonas desfavorecidas, Madrid, Consejo Económico y Social.

Cruz Ferrer, J. (1999) La liberalización de los servicios públicos y el sector eléctrico. Modelos y análisis de la Ley 54/1997, Madrid, Marcial Pons.

Díaz de LA Rosa, A. (2007) Las cooperativas de iniciativa social, Anuario de la Facultad de Derecho de la Universidad de La Coruña, N. ${ }^{\circ} 11$, p. 201-210.

EMBID IRUjo, A. (1991), La planificación hidrológica: régimen jurídico, Madrid, Tecnos.

ForshofF; E. (1967) Sociedad industrial y Administración Pública, Madrid, Escuela Nacional de Administración Pública.

GADEA Soler, E. (2009) Estudio sobre el concepto de cooperativas: referencia a los principios cooperativos y su discutida vigencia, JADO, Boletín de la Academia Vasca de Derecho, N. ${ }^{\circ} 17$, p. 165-185.

Garcés Sanagustín, A. (1996) Prestaciones sociales, financiación administrativa y derechos de los ciudadanos, Madrid, McGraw Hill.

García de EnterRía, E. (1998) Democracia, jueces y control de la Administración, Madrid, Civitas, $4 .^{\text {a }}$ ed.

García MACHO, R. (2011) La transparencia en el sector público, en El derecho público de la crisis económica. Transparencia y sector público. Hacía un nuevo Derecho Administrativo, Madrid, Instituto Nacional de Administración Pública.

García Ruiz, E. (2013) El negocio fundacional en las sociedades cooperativas de participación pública, REVESCO. Revista de Estudios Cooperativos, 2013, núm. 111, p. 60-89.

HeRnÁNDEZ, J.C. (2005) Regulación y competencia en el sector eléctrico. Evolución, regulación actual y perspectivas de futuro, Pamplona, ThomsonAranzadi.

Jiménez Escobar, J. y Morales Gutiérrez, A.C. (2006) Dirección de entidades no lucrativas. Marco jurídico, análisis estratégico y gestión, Pamplona, Aranzadi, 2006.

Martín Rebollo, L. (1984) El Derecho administrativo y las relaciones entre las Administraciones Públicas y los ciudadanos. Lección de apertura del Curso académico 1984-1985, Cáceres, Universidad de Extremadura. 
Martín Rebollo, L. (1983) Estado social y Administración Pública. Los postulados constitucionales de la reforma administrativa, Madrid, Civitas.

Martín-Retortillo Baquer, S. (1992), en el Prólogo del libro de Sánchez Blanco, A. El sistema económico de la Constitución (participación institucional de las Autonomías territoriales y dinámica social en la economía), Madrid, Civitas.

Martín-Retortillo Baquer, S. (2009) De la simplificación de la Administración Pública, Revista de Administración Pública, N.ำ17, p. 165-185.

MIR PUIG, O (2004). Globalización, Estado y Derecho. Las transformaciones recientes del Derecho administrativo, Madrid, Thomson-Civitas.

Molina Aparicio, F. (2009) Historia del Consejo Superior de Cooperativas de Euskadi, Vitoria-Gasteiz, Consejo Superior de Cooperativas de Euskadi.

Montero Simó, M. (2010) La fiscalidad de las cooperativas sin ánimo de lucro, CIRIEC-España. Revista de Economía Pública, Social y Cooperativa, N. ${ }^{\circ} 69$, p. 161-190.

Nebreda Pérez, J.M. (2003), Distribución eléctrica. Concurrencia de disciplinas jurídicas, Madrid, Thomson-Aranzadi, 2. ${ }^{\text {a ed. }}$

Palomar Olmeda, A. (2010) Simplificación administrativa, en La ordenación de las actividades de servicios: comentarios a la Ley 17/2009, de 23 de noviembre, Quadra-Salcedo Fernández Castillo, T. (dir), Pamplona, Aranzadi, p. 331-362.

Ruíz Olabuénaga, J.L. (2006) El sector no lucrativo en España. Una visión reciente, Bilbao, Fundación BBVA.

SALAS, A. (2009) El tercer sector en España, en Gestión actual de una ONG, Moro, L. (coord.), Madrid, LID editorial empresarial, p. 29-42.

VARGAS VASSEROT, C. (2012) La organización de entidades público-privadas a través de sociedades mercantiles en El gobierno y la gestión de las entidades no lucrativas público-privadas, EMBID IRUjo, J.M. y Emparanza Sobejano, A. (Dirs.), Madrid, Marcial Pons, p. 93-135.

Zurbano, M., Henry, G y Urzelal, A. (2012) El cuarto sector en Euskadi, Zamudio, Innobasque. 